\title{
Démarche qualité en médecine: un état des lieux par portraits succincts (12)
}

www fmh $\mathrm{ch} \rightarrow$ Welcome $\rightarrow$ Qui sommes-nous? $\rightarrow$ Le Secrétariat général $\rightarrow$ Données, démographie \& qualité
La démarche qualité en médecine s'est établie dans notre pays. C'est ce que le groupe de travail Qualité de la FMH (GTQ-FMH) tient à montrer aux membres de la FMH. Il présente ainsi dans cinq numéros consécutifs du BMS les initiatives Qualité qui lui ont été annoncées depuis 2006 par des médecins de toutes les disciplines médicales et de tous les secteurs d'activité (domaine hospitalier/ambulatoire). Ces descriptifs sont également publiés sur le site internet de la FMH, Département Données, démographie et qualité avec des informations pratiques telles que graphiques ou bibliographie.* siris, le registre suisses des implants, est présenté ici.

\section{siris - Registre suisse des implants}

Pour le lecteur pressé

Le Registre suisse des implants (siris)

... recueille et centralise l'ensemble des données relatives aux endoprothèses (articulations) de la hanche et du genou

... procède à la saisie d'un corpus minimal de données relatives à un implant englobant les éléments relatifs à la pathologie, la chirurgie, de fixation et de type d'implant de même que le suivi du traitement: des questions complémentaires pour d'autres études sont possibles

... livre des données comparables aux hôpitaux participants. L'industrie, les caisses-maladie et les tiers ne reçoivent que des données anonymisées, dont la publication a été autorisée au préalable par le Conseil de fondation.

\section{Description}

siris procède à la saisie d'un corpus minimal de données relatives aux endoprothèses (articulations) de la hanche et du genou englobant les aspects relatifs à la pathologie, la chirurgie, la fixation et le type d'implant (au moyen d'un scanner à codes-barres), de même que le suivi du traitement. Les collaboratrices et collaborateurs concernés peuvent remplir en ligne le set de données; compte tenu de la clarté de sa présentation, des connaissances spécifiques ne sont pas forcément requises.

Le corps de données de base peut être élargi à des questions complémentaires spécifiques et peut être relié à des études existantes. Sous réserve de son accord, le numéro AVS du patient peut être saisi au sein de siris, permettant des observations sur le long terme.
Le Registre suisse des implants siris est subordonné au conseil de fondation, qui compte des représentants des organisations suivantes: Société suisse d'orthopédie (SSO), santésuisse, H+ et la Fédération des associations suisses du commerce et de l'industrie de la technologie médicale (FASMED), cette dernière association étant exclusivement représentée par le biais des fabricants d'implants orthopédiques. Le conseil de fondation est le dépositaire des données siris.

Les hôpitaux qui participent à siris reçoivent des données comparatives qui situent la position des chirurgiennes et des chirurgiens de leur hôpital resp. de leur région ou pays mais aussi celle des autres hôpitaux de la région ou du pays. L'industrie, les caisses-maladie et les tiers ne reçoivent de données que sous une forme anonymisée et après autorisation de publication par le conseil de fondation.

\section{Aptitude}

Hôpitaux, médecins agréés

\section{Charges et coûts}

Temps nécessaire: par cas environ 5 minutes. Charge financière: par implant, il est facturé CHF 35.par cas. Il s'agit de coûts imputables, qu'il sera possible de faire valoir lors des prochaines négociations contractuelles avec les caisses-maladie. Les hôpitaux qui facturent séparément les implants peuvent immédiatement facturer les charges relatives aux caisses.

\section{Informations complémentaires}

www.siris-implant.ch
Fax 0316315960

urs.mueller@

memcenter.unibe.ch 